\title{
A BIO-INSPIRED SOLUTION FOR MANUFACTURING CONTROL SYSTEMS
}

\author{
Paulo Leitão \\ Polytechnic Institute of Bragança, Quinta Sta Apolónia, Apartado 1134, 5301-857 Bragança, \\ Portugal,pleitao@ipb.pt
}

\begin{abstract}
Manufacturing is nowadays facing with markets trends that ask for more customized products, shorter product life-cycles, best quality and shorter prices. Addressing these requirements, manufacturing systems need to be more responsive and reconfigurable, adapting their behaviors to changing conditions. The concepts inherited from biology and nature seem suitable to design reconfigurable manufacturing systems. In this paper, a bio-inspired solution, where self-organization and multi-agent systems play key roles, is described, contributing to achieve an adaptive and evolvable reconfigurable manufacturing control system.
\end{abstract}

\section{INTRODUCTION}

Cost, quality and responsiveness are the three foundations on which every manufacturing company stands, to be competitive in the current global economy (ElMaraghy, 2006). Increasingly, traditional centralized and sequential manufacturing systems are being found insufficiently flexible to respond to changing production style and highly dynamic variations in production requirements. Under these circumstances, companies are forced to have manufacturing systems that exhibit innovative features to support the agile response to the emergence and changing conditions by the dynamic re-configuration on fly, i.e. without stopping, re-programming or re-starting the process. Briefly, reconfigurability can be defined as the ability to repeatedly change and re-arrange the components of a system in a cost-effective way (Setchi et al., 2004). In manufacturing domain, reconfiguration implies a change in the control software or hardware of industrial automation and control systems, allowing the shop floor to adapt automatically to change while maintaining predictable and stable system behavior. Typically, a change would be required if the system is upgraded (e.g. new software components, hardware drivers or the hardware itself) or as a contingency to some event (e.g. the failure of a piece of hardware or a systematic error in the software). Re-configurability plays a key role in the new generation of production control systems, providing a way to achieve a rapid and adaptive response to change, which is a key enabler of competitiveness.

This idea is reinforced by two studies, one elaborated by the US Committee on Visionary Manufacturing (CMV, 1998) and another sponsored by the European Commission (Manufuture, 2004), which have identified reconfigurable manufacturing as the highest priority for future research in manufacturing. In fact, the first study states that the reconfigurable manufacturing is one of the six key manufacturing challenges for the year 2020, and the second one points out the need to have adaptive, digital, networked and knowledge-based manufacturing processes. They propose that manufacturing processes, 
components and systems have to be re-adaptable and re-configurable for a wide range of customer requirements for products, features and services.

Reconfigurable Manufacturing Systems (RMS) are "designed at the outset for the rapid change in its structure, as well as its hardware and software components, in order to quickly adjust its production capacity and functionality within a part family in response to sudden market changes or intrinsic system change" (Koren et al., 1999). RMS possesses characteristics of modularity, integrability, customization, scalability, convertibility and diagnosability (ElMaraghy, 2006), which impose, among others, strong requirements to the control solution, with manufacturing systems built on centralized structures becoming unsustainable due to its intrinsic rigidity. These characteristics can be applied to the design of whole manufacturing systems, as well as to some of its components, i.e. reconfigurable machines, their controllers and also to the control system software. A typical RMS has several of these characteristics but not necessarily all.

The concept of RMS is a step ahead of the concept of Flexible Manufacturing Systems (FMS), presenting different goals. FMS aims at increasing the variety of parts produced, and RMS aims at increasing the speed of responsiveness to changing conditions. RMS instead of incorporating all the flexibility once at the beginning of their life-cycle should incorporate basic process models - both hardware and software - that can be rearranged or replaced quickly and reliably (Mehrabi et al., 2000). In other words, in a FMS it is necessary to install in the beginning all functionalities that can be used in the future, while in a RMS the functionalities are added during its life-cycle according to the needs (Mehrabi et al., 2000). Biology and nature are suitable sources of inspiration for the development of RMS, mainly addressing the development of adaptive and evolvable systems. For this purpose, some theories inherited from biological systems, such as artificial life, chaos theory, swarm intelligence and complexity behavior may be applied. However, at the time, significant proofs about the applicability of reconfigurable solutions in industrial environments are missing, since only very few implementations of reconfigurable solutions have been reported in the literature. Additionally, the implemented functionalities are normally restricted. This requires the implementation of bio-inspired solutions in real scenarios to proof its real applicability. This paper illustrates the applicability of bio-inspired theories to build reconfigurable manufacturing (control) systems. In this context, a bio-inspired solution is discussed, presenting evolving mechanisms based on self-organization, supervision and learning concepts and on antbased communication, supported by the use of multi-agent principles.

The paper is organized as follows: first, Section 2 overviews the concept of selforganization and how it can be applied in manufacturing systems. Section 3 discusses the foundations of a bio-inspired solution for manufacturing control and Section 4 describes the adaptive control system working in practice by presenting how the control structure evolves dynamically and how the task allocation is performed. Finally, Section 5 rounds up the paper with the conclusions. 


\section{APPLYING SELF-ORGANIZATION TO MANUFACTURING SYSTEMS}

Fundamental works to provide control architectures to solve RMS requirements are Multiagent Systems (Wooldridge, 2002) and Holonic Manufacturing Systems (HMS) (Koestler, 1969), (Brussle et al., 1998), (Deen, 2003). Multi-agent systems, derived from distributed artificial intelligence, suggest the definition of distributed control based on autonomous agents that account for the realization of efficient, flexible and robust overall plant control, and consequently the disturbance handling component. In a similar way, HMS translates into the manufacturing world the concepts developed by A. Koestler for living organisms and social organizations. Holonic manufacturing is characterized by holarchies of holons (i.e., autonomous and cooperative entities), which represent the entire range of manufacturing entities. A holon, as Koestler devised the term, is a part of a (manufacturing) system that may be made up of sub-ordinate parts and, in turn, can be part of a larger whole.

The capability of adaptation and evolution to face emergence, crucial in RMS, requires the implementation of complex adaptive algorithms within distributed control approaches, e.g. the MAS and HMS. A suitable approach is to translate some mechanisms and concepts founded in the biology and nature to build adaptive reconfigurable systems. In fact, biology and nature offer powerful mechanisms built on entities that exhibit simple behaviors and have limited cognitive abilities, where a small number of rules or laws can generate systems of surprising complexity (Holland, 1998). Complex behavior may then emerge from interactions among entities exhibiting simple behavior (Bonabeau et al., 1999), being the behavior of the whole much more complex than the behavior of the parts (Holland, 1998). The emergence only occurs when the resulted whole is more than the sum of its parts (Holland, 1998).

The swarm intelligence concept, also inherited from biology, can be defined as the emergent collective intelligence of groups of simple and single entities. It offers an alternative way of designing intelligent systems, in which autonomy, emergence, and distributed functioning replace control, pre-programming and centralization (Bonabeau et al., 1999). As an example, a social insect colony is a decentralized system composed of cooperative, autonomous units that are distributed in the environment, exhibit simple probabilistic stimulus-response behavior, and have access to local information (Bonabeau et al., 1999). The system exhibiting these characteristics operates in a very flexible and robust way (Bonabeau et al., 1999), where flexibility allows adaptation to changing environments and robustness endows the colony with the ability to work even if some individuals may fail to perform their tasks.

In biological systems there are two different approaches to adaptation to the dynamic evolution of the environment (Vaario et al., 1996): evolutionary systems and selforganization. In the evolutionary approach, the nodes of the control structure are encoded as genetic information and are subject to the application of evolutionary techniques by selecting gradually a better system. Well known examples of this approach are neural networks, where learning occurs in an evolutionary way, and genetic algorithms, where problems are solved by an evolutionary process. In the self-organizing approach, the network of nodes that represents the control system is established by the nodes themselves. The driving forces drive the re-organization process according to the environment conditions and to the control properties of the distributed entities. In other words, the self-organization is viewed when the evolution is determined by the reorganization of the structure through the addition/removal of the relationships among 
entities and through the addition/removal of the goal-oriented activities lead by the entities.

Self-organization is not a new concept, being applied in different domains such as computing and robotics. Several distinct definitions, but not necessarily contradictory, are found in the literature. The Intelligent Manufacturing Systems (IMS) consortium defines a self-organized system as a system which is not coordinated or regulated by the exterior, and Massote (Massotte, 1995) defines self-organization as the integration of autonomy and learning capabilities within entities to achieve, by emergence, global behavior that is not programmed or defined a priori. Thamarajah (Thamarajah, 1998) defines selforganization as the ability of an entity/system to adapt itself to prevailing conditions of its environment and Bousbia and Trentesaux (Bousbia and Trentesaux, 2006) defines selforganization as the ability of an entity to look after and ensure its optimal functioning with minimum help or intervention by external or internal component of the system.

A possible way to integrate self-organization capabilities is to move from fixed and centralized architectures to distributed ones (Bousbia and Trentesaux, 2006), that does not follow a rigid and estimated organization. In fact, autonomous systems, as our brain, have to constantly optimize their behavior, involving the combination of non-linear and dynamic processes. These characteristics imply the management and control of behavioral complexity as well. The application of self-organization allows the dynamic selfconfiguration (i.e. adaptation to changing conditions by changing their own configuration permitting the addition/removal of resources on fly and without service disruption), selfoptimization (i.e. tuning itself in a pro-active way to respond to environmental stimuli) and self-healing (i.e. capacity to diagnose deviations from normal conditions and take proactive actions to normalize them and avoid service disruptions).

The self-organization of each distributed entity can be defined as the capability to organize by itself into different structures, according to its perception of the environment. The emergence of the global control or organizational structure is a result of the capability of local entities to change dynamically and autonomously their properties. In such system, the network of control entities is coordinated towards a unique goal or objective to evolve, with a new solution for the re-organization achieved by emergence. During the reorganization process it is necessary to evaluate, according to a specific criteria, if the achieved solution is better than the previous one (Pujo et al., 2001).

When dealing with reconfigurable systems, in which distribution and emergence play key roles, it is crucial to have regulation mechanisms to introduce order and stability, avoiding the increase of entropy and consequently the chaotic or instable states. Here, it is important to remark the analogy with the 2 nd Law of Thermodynamics that states that the total entropy of any isolated thermodynamic system tends to increase over the time, approaching a maximum value.

Self-organization can contribute to adaptive manufacturing systems in the main following areas (Vaario et al., 1997):

- Shop floor layout, where the manufacturing entities present in the shop floor are movable, e.g. to minimize the transportation distances;

- Adaptive control, in which the goal is to find out an adaptive and dynamic production control strategy based in the dynamic and on-line schedule, adapted in case of occurrence of unexpected disturbances;

- Product demand, in which the manufacturing system re-organizes itself in order to adapt to the changes in the product demand, increasing or reducing the number of manufacturing resources, or modifying their capabilities. 
The work presented in this paper focuses the adaptive control, with self-organization contributing for the dynamic re-organization of the control system.

\section{BIOLOGICAL-INSPIRED CONTROL SOLUTION}

The Darwin's theory of the evolution of species tell us that species change over a long period of time, evolving to suit their environment, and that the species that survive to changes in the environment are not the strongest or the most intelligent, but those that are more responsiveness to change. Translating into the manufacturing domain, the manufacturing companies better prepared to survive are those that better respond to emergent and volatile environments.

\subsection{Basic Concepts}

Having this idea in mind, the ADACOR (ADAptive holonic COntrol aRchitecture for distributed manufacturing systems) holonic manufacturing control architecture introduces an adaptive and evolvable approach, considering bio-inspired theories, that addresses the system re-configurability and evolution, especially in emergent environments. The proposed adaptive architecture intends to be as decentralized as possible and as centralized as necessary, i.e. evolving from a centralized approach when the objective is the optimization, to a more heterarchical approach in presence of unexpected scenarios.

ADACOR architecture is based on concepts inherited from biology, social organizations and artificial life, namely self-organization and swarm intelligence, translating into the manufacturing world the way how complex biological entities and systems behave in a simple way. It is based on the following main foundations: holonic manufacturing principles, supervisor entities and self-organization.

In analogy to insect colonies, ADACOR architecture is built upon a community of autonomous and cooperative holons, representing the manufacturing components, such as robots, conveyors, pallets, products and orders. Using HMS principles, the manufacturing control functions are distributed among holons, taking advantage of modularity, decentralization, agility, flexibility, robustness and scalability.

In social society colony, an individual usually does not perform all tasks, but rather specializes in a set of tasks (Bonabeau et al., 1999). In fact, the division of labor is used to have specialized individuals performing simultaneously different activities, which is believed to be more efficient than sequential task performance by unspecialized individuals (Bonabeau et al., 1999). Following this idea, ADACOR architecture identifies four manufacturing holon classes (Leitao, Restivo, 2006), product ( $\mathrm{PH})$, task (TH), operational $(\mathrm{OH})$ and supervisor $(\mathrm{SH})$, according to their roles and functionalities. The product holons represent the products (and sub-products) available in the factory catalogue, containing all knowledge related to the product. The task holons represent the production orders launched to the shop floor to execute the requested products and the operational holons represent the physical resources available at shop floor, such as operators, robots and conveyors. Operational holons are also specialized to perform physical tasks, namely machining and moving. The supervisor holons represent the logical coordination of a group of holons, providing co-ordination and optimization services to the holons under their supervision, and thus introducing hierarchy in an otherwise decentralized system. 
The product, task and operational holons are quite similar to the product, order and resource holons defined in PROSA reference architecture (Brussel et al., 1998), while the supervisor holon, inspired in biological systems, presents characteristics not found in the PROSA staff holon, namely the possibility to coordinate other supervisor holons in a federation architecture and the responsibility to manage the dynamic evolution of groups of holons according to the environment context (Leitao, Restivo, 2008). ADACOR holons are of the plug and produce type, being possible to add a new element without the need to re-initialize and re-program the system, thus allowing high flexibility in system adaptation and re-configuration on fly.

The self-organization exhibited by each distributed holon allows the dynamic evolution and re-configuration of the organizational control structure, combining the global production optimization with the agile reaction to unexpected disturbances. As in biological systems, where the evolution of the species or the groups results from the selforganization of local entities, the adaptive ADACOR mechanism, as illustrated in Figure 1, emerges from a bottom-up approach, built upon the individual self-organization of manufacturing holons. Here, the dynamic adaptation of each holon to unexpected situations contributes to the adaptation of the system as a whole to the emergent contexts and to the quick reaction to the occurrence of unexpected disturbances.

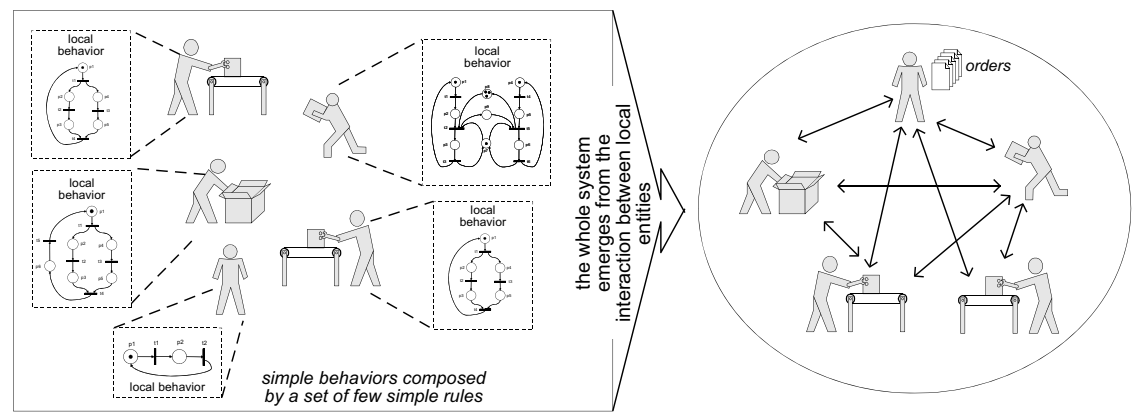

Figure 1. Global System Emerged from the Behavior of Local Holons

\subsection{Individual Driving forces}

The self-organization mechanism requires local driving forces to support the adaptation. In ADACOR architecture, the driving forces are the autonomy factor and the learning capability, which are inherent characteristics to each ADACOR holon.

An ADACOR holon is autonomous, since it can operate on its own, without the direct intervention of external entities, and has full control over its behavior. Having its own objectives, knowledge and skills, each holon has the capability to reason in order to take decisions about its activities. Each ADACOR holon possesses only a partial view of the system, needing to cooperate with the other holons in order to achieve its goals or to get additional information about the system.

Aiming to achieve an adaptive and evolvable behavior, ADACOR introduces the autonomy factor concept, designated by $\alpha$, that is a parameter associated to each holon reflecting its degree of autonomy (Leitao, Restivo, 2006). The autonomy factor is regulated by a decision mechanism that evolves dynamically in order to adapt the holon 
behavior to changes in the environment where it is placed. The evolution of the autonomy factor is regulated by a fuzzy rule-based engine, illustrated in

Figure 112, which takes into consideration the reestablishment time $(\tau)$, which is the estimated time to recover from the current disturbance, and the pheromone parameter $(\rho)$, which is an indication of the level of impact of the disturbance.

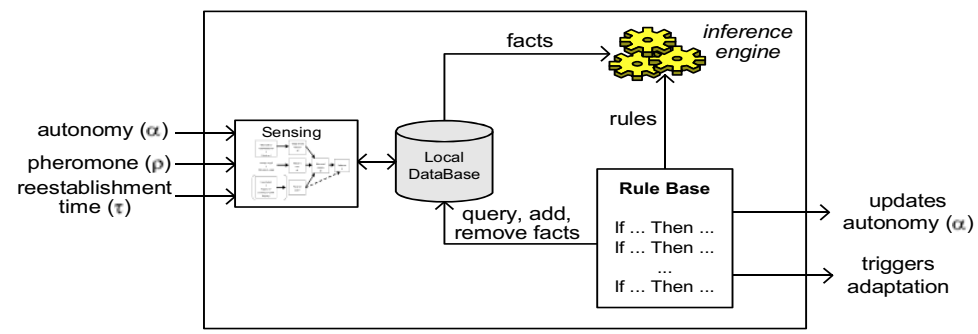

Figure 2. Rule-based Mechanism to Regulate the Holon's Autonomy

The cardinality of the numerical set associated to the autonomy factor may have strong impact in the dynamic of the adaptation mechanism: on one hand, the higher the number of values, the more gradual will be the adaptation procedure, but, on the other side, a high number of values makes the adaptation mechanism more complex and the response times longer (Leitao, Restivo, 2006). In this study, it was considered that the autonomy factor is a discrete binary variable comprising the states \{Low, High $\}$.

The set of simple rules that regulates the adaptation behavior of the holon is illustrated in Figure 3. Normally, the operational holons have a low autonomy factor, following the supervisor holon coordination and accepting its schedule proposals. In case of $\{$ Low $\}$ autonomy factor, the emergency, normally the occurrence of a disturbance and represented by the $\{$ High $\}$ value associated to the pheromone parameter $(\rho)$, triggers the change of the autonomy factor to $\{$ High $\}$ and the re-organization process.

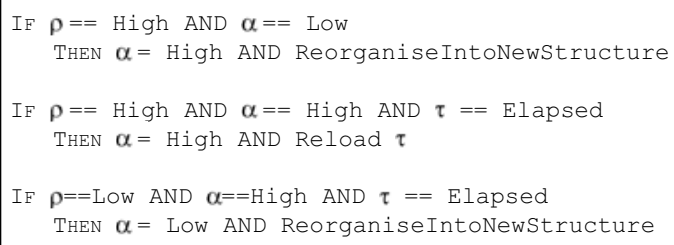

Figure 3. Set of Simple Rules that Regulates the Adaptive Mechanism Behavior

When the reestablishment time elapses, if the autonomy factor is $\{$ High $\}$ and the pheromone is still active, which means that the disturbance is not completely recovered, the reestablishment time is re-loaded. If the pheromone has already dissipated, which means that the disturbance is already solved, the holon can return to the original structure, changing the autonomy factor to $\{$ Low $\}$.

The degree of efficiency of the self-organization capability, and consequently the improvement of the holon's behavior performance supporting its adaptation to the environment emergency, is strongly dependent on how the learning mechanisms are implemented. The learning capability associated to ADACOR holons allows the acquisition of new knowledge, improving the holon's ability to act in future and to 
support the dynamic evolution of the environment where it is placed. The learning capability is dependent on the decision mechanisms and on the learning algorithms. As an example, in case of neural networks, the learning is associated with the adjustment of the nodes coefficients, but in case of expert systems, the learning is associated with the addition of new facts or to the generation of new rules. The elaboration of new rules is more complex than the simple acquisition of new factual knowledge, and requires a special attention to verify dynamically the possible contradiction between the new knowledge rules and the initial behavior knowledge.

\subsection{Global Driving Forces to Achieve Evolvable Systems}

The driving forces associated to each individual entity aiming to achieve adaptation were analyzed in the previous section. The global self-organization of the system is only achieved if the distributed entities have stimulus to drive their local self-organization capabilities. The behavior recalls the stimergy concept, which is often used in biology to describe the influence on behavior of the persisting environmental effects of previous behaviors.

The global self-organization requires global mechanisms that allow the interaction between local individual holons, supporting the propagation of the emergence, which constitutes the event-driven mechanism to trigger the evolution of the control system into different structures. ADACOR approach proposes a pheromone-like spreading mechanism to propagate the emergence, triggering the evolution process. The entities cooperating with this type of mechanisms (Brussel et al., 2000):

- Dissipate the information to the other entities, in a similar way to the ant that deposits pheromones;

- Sense the information dissipated by the other entities (like ants sense the odors) to take their own actions; sometimes they reinforce the odor.

In the ADACOR architecture, when an emergence or an unexpected disturbance occurs, the need for re-organization is propagated through the deposit of a certain quantity of pheromone in the neighbor supervisor holons, as illustrated in Figure4. This quantity is proportional to the estimated reestablishment time, forecasted according to the type of disturbance and to the historic data.

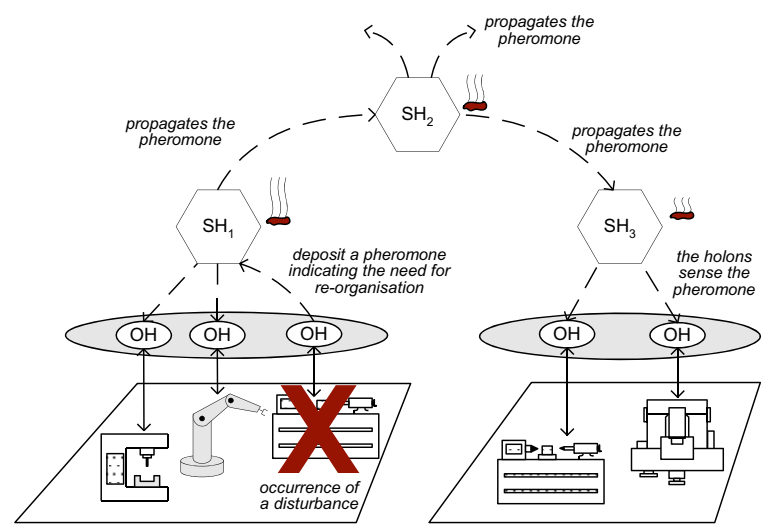

Figure 4. Propagation of the Emergence using Pheromone-like Techniques

The holons associated to each supervisor holon receive the need for re-organization by sensing the pheromone and propagating this need to neighbor holons. The intensity of the 
odor associated to the pheromone becomes smaller with the increase of the number of the levels of supervisor holons (similar to distance in the original pheromone techniques), according to a defined flow field gradient, which is characterized by the reduction of the intensity of the odor and increase of the entropy. With this mechanism, holons positioned near of the disturbance epicenter will be more sensitive to the self-evolution than the holons positioned far from the epicenter. While the emergence is still active, e.g. because the disturbance was not completely solved, the holon reinforces the odor associated with the pheromone indicating that the problem still remains. The holon stops to reinforces the odor when the problem is solved.

The propagation of the emergence and the need for re-organization using pheromonelike techniques is suitable for the dynamic and continuous adaptation of the system to disturbances, supporting the global self-organization, reducing the communication overhead and improving the reaction to disturbances (Leitao, Restivo, 2006).

\section{ADAPTIVE CONTROL WORKING IN PRACTICE}

The control architecture is the key factor for the performance of the manufacturing control system, playing a critical role in the system performance in terms of response to change and capability to learn. The proposed dynamic and adaptive control approach improves the agility and reaction to unexpected disturbances without compromising the global optimization. In this section, the adaptive control approach will be further analyzed, mainly the dynamic evolution of the control structure and the task allocation mechanism.

\subsection{Dynamic Evolution of the Control Structure}

The ADACOR adaptive control balances between a more centralized approach and a more flat approach, passing through other intermediate forms of control (Leitao, Restivo, 2006), due to the adaptive and dynamic evolution of the autonomy factor of each ADACOR holon. The control is shared between supervisor and operational holons, and is splited into two alternative states (Leitao, Restivo, 2006): a stationary state, in which the system control uses coordination levels to get global optimization of the production plan, and a transient state, triggered by the occurrence of disturbances and presenting a behavior quite similar to the heterarchical approach in terms of agility and adaptability.

In stationary state the holons are organized in a hierarchical architecture, with supervisor holons interacting directly with the task holons during the task allocation process. Supervisor holons, as coordinators, elaborate optimized schedule plans that are proposed to the task holons and to the operational holons under their coordination domain. In this state, each operational holon has a low autonomy factor and sees these proposals as advices, following the proposals, although they have enough autonomy to accept or reject the proposed schedule (Leitao, Restivo, 2006).

If, for any reason, the system deviates from planned, due for example to a machine failure or a rush order, the operational holon which detects the disturbance increases its autonomy factor parameter and propagates the need for re-organization to the other holons in the system using pheromone-like techniques. The neighbor holons also sense the pheromone and will increase their autonomy factors according to the intensity of the pheromone and their local knowledge, entering in a transient state. In this state the holons evolve to a new control structure aiming to achieve an agile reaction to the emergency, with the self-organization capability playing a crucial role. The task holons interact directly with the operational holons in order to achieve an alternative schedule plan and 
the supervisor holons can continue elaborating and proposing the allocation of operations to the operational holons, but since these have now high values of autonomy factors, they will probably reject the proposals (Leitao, Restivo, 2006). The holons remain in the transient state during the reestablishment time, $\tau$, which is typically a short period of time estimated by the operational holon that detected the disturbance for its recovery.

After the recovery from the disturbance, the holon ends the reinforcement of the pheromone. When the other holons don't sense the pheromone odor anymore, they reduce their autonomy factors and the system evolves to a new control structure (often returning to the original one), according to the learning capabilities embedded in each holon. The supervisor holon returns to its coordination function, re-scheduling the non-optimized schedule, reached during the transient state.

\subsection{Task Allocation}

A result of the proposed dynamic evolving mechanism is the presence of a scheduling approach that distributes the scheduling functions over several entities, combining their calculation power and local optimization. In this scheduling approach, the objective is to achieve fast and dynamic re-scheduling using a scheduling mechanism that evolves dynamically to combine centralized strategies and distributed strategies, improving its responsiveness to emergence, instead of the complex and optimized scheduling algorithms. The idea is that a global optimized schedule should be generated whenever possible, and a fast re-scheduling should be used in case of disturbances, because, in this case, this is preferable than waiting a significant amount of time for an optimized schedule, which is likely to be not optimized again soon (Leitao, Restivo, 2008).

In the distributed scheduling model the computational complexity is related to find an optimal determination problem in combinatorial auctions, with the presence of two specialized types of holons: task holons that have operations to be executed and operational holons that have skills to execute operations. The motivation of ADACOR holons to execute the manufacturing control functions is regulated by a credits system. Table 1 summarizes the evolution of the credits of task and operational holons during their life cycles.

Table 1. Evolution of Credits During the Holon's Life Cycle

\begin{tabular}{|l|l|l|}
\hline Phase & Task Holon & Operational Holon \\
\hline Initially. & $\pi$ credits to execute the order & None credits \\
\hline $\begin{array}{l}\text { Operation allocation } \\
\text { process. }\end{array}$ & $\begin{array}{l}\text { Contracts the operation execution by } \varphi \\
\text { and the penalty by } \xi .\end{array}$ & $\begin{array}{l}\text { Contracts the operation execution } \\
\text { by } \varphi \text { and the penalty by } \xi .\end{array}$ \\
\hline $\begin{array}{l}\text { End of an operation } \\
\text { with success. }\end{array}$ & Pays the value $\varphi$ to the $\mathrm{OH}(\pi \leftarrow \pi-\varphi)$. & $\begin{array}{l}\text { Increases the total credits by } \varphi \\
(\mu \leftarrow \mu+\varphi) .\end{array}$ \\
\hline $\begin{array}{l}\text { End of an operation } \\
\text { with delay. }\end{array}$ & $\begin{array}{l}\text { Pays the value } \varphi \text { and receives the value } \\
\xi \text { from the } \mathrm{OH}(\pi \leftarrow \pi+\xi-\varphi) .\end{array}$ & $\begin{array}{l}\text { Decreases the total credits by } \xi \\
\text { and increase by } \varphi(\mu \leftarrow \mu-\xi+\varphi) .\end{array}$ \\
\hline $\begin{array}{l}\text { Operation cancelled } \\
\text { (delay, failure, etc.). }\end{array}$ & $\begin{array}{l}\text { Receives the value } \xi \text { from the } \mathrm{OH} \\
(\pi \leftarrow \pi+\xi) .\end{array}$ & $\begin{array}{l}\text { Decreases the total credits by } \xi \\
(\mu \leftarrow \mu-\xi) .\end{array}$ \\
\hline
\end{tabular}

When the task holon is launched, it receives a fund to execute the production order $(\pi)$ and a penalty value for delay. The task holon manages the costs to execute its production order in order to guarantee that they never exceed the initial fund. During the interaction to allocate the operations, the task holons try to pay as less as possible and the operational holons try to receive as more as possible.

Initially, the task holons announce, in an open market basis, the execution of the operations belonging to the order. In presence of operation announcements, each 
operational holon decides, based on its skills and capacity, its availability to execute the operation. Since each operation has a set of requirements, each operational holon verifies if it can perform the operation by matching the requirements of the operation with the resource's skills, using a rule-based function. In case of availability, the operational holon calculates the price to be proposed to the task holon, $\mathrm{p}_{\mathrm{jik}}$, that may be calculated according to the following function:

$$
p_{j i k}=C_{s}+C_{p} \times d_{i k}+C_{b} \times\left(-e^{-\sigma \times \beta} \times\left(-\gamma^{2}\right)\right.
$$

where $C_{s}$ is the cost associated to the setup execution, $C_{p}$ is related to the cost associated to maintain the machine working, $\mathrm{d}_{\mathrm{ik}}$ is the duration of the operation execution and $\mathrm{C}_{\mathrm{b}}$ reflects the investment done to buy the machine. In order to have a dynamic price, this expression models the market laws, increasing or decreasing the final price in function of the actual load of the resource (reflected by the parameter $\beta$ ) and of the actual bid acceptance rate (reflected by the $\gamma$ parameter, with $0 \leq \gamma \leq 1$ ). The operational holon uses the knowledge learned from the previous bids to adjust the final price: decreasing the $\gamma$ parameter if the acceptance rate is low or increasing it in the opposite case.

The decision to select the best proposal, taken by the task holon, is achieved by minimizing a heuristic function that takes in consideration, among others, the proposed price, the location of the resource and the confidence degree of the proponent operational holon. The confidence degree reflects the trust that the task holon has in an operational holon and is based on the knowledge learned in previous interactions, considering a percentage of operations successfully executed by the resource. In case of an inconclusive evaluation, the task holon can start another iterative negotiation, re-formulating the bid parameters, for example the due date and announcement specifications. After the negotiation, the task holon accepts to pay a price of $\varphi$ credits (i.e. $p_{j i k}$ ) to the operational holon that will execute the operation and to receive a penalty of $\xi$ credits from the operational holon if it does not fulfill the contracted due date.

The global performance of the operational holons in terms of credits is given by the sum of rewards received minus the penalties paid for the delays.

\section{CONCLUSIONS}

Reconfigurable manufacturing systems appear as an emergent paradigm to face the challenge for agile, adaptive and evolvable systems, where re-configurability and responsiveness play key roles. In fact, these systems should exhibit agile response to emergence by providing dynamic re-configuration on fly, i.e. without stopping, reprogramming or re-starting the process. Biological and nature inspired concepts and theories, namely artificial life, chaos theory, swarm intelligence and complexity behavior, seem suitable for the design of reconfigurable manufacturing systems.

This paper illustrates the applicability of these concepts in manufacturing world by introducing a bio-inspired solution for reconfigurable manufacturing control systems, using concepts derived from holonic manufacturing, swarm intelligence and selforganization. The proposed approach introduces an adaptive control approach based on self-organization, supervision and learning concepts and on ant-based communication, supported by the use of multi-agent principles.

The preliminary experimental results have proved the applicability of the proposed bio-inspired solution (Leitao, Restivo, 2006). Future work is related to the introduction of powerful biological and intelligence mechanisms to support better the dynamic re- 
configurability of the control system, namely learning mechanisms to identify the situations and the way to evolve.

\section{REFERENCES}

1. Bonabeau, E., Dorigo, M and Theraulaz, G., "Swarm Intelligence: From Natural to Artificial Systems", Oxford University Press, 1999.

2. Bousbia, S., Trentesaux, D., "Self-organization in Distributed Manufacturing Control: State-of-the-art and Future Trends", Proc. of the International Conference on Systems, Man and Cybernetics, vol5, 2002.

3. Brussel, H.V., Valckenaers, P., Wyns, J., Peeters, P. and Bongaerts, L., "Holonic Manufacturing Systems, Architectural and Manufacturing Control Issues", Proceedings of 2nd CIRP Int'1 Seminar on Intelligent Computation in Manufacturing Engineering, 2000, pp. 19-29.

4. Brussel, H.V., Wyns, J., Valckenaers, P., Bongaerts, L. Reference Architecture for Holonic Manufacturing Systems: PROSA. Computers In Industry 1998; 37(3): 255-274.

5. CMV, "Visionary Manufacturing Challenges for 2020", Committee on Visionary Manufacturing, 1998, National Academic Press: Washington DC, USA.

6. Deen, S. (editor), Agent-Based Manufacturing: Advances in the Holonic Approach, Springer Verlag Berlin Heidelberg, 2003.

7. ElMaraghy, H. Flexible and Reconfigurable Manufacturing Systems Paradigms. International Journal of Flexible Manufacturing Systems, 17, 2006, pp. 261-271.

8. Holland, J., "Emergence: from Chaos to Order", Oxford University Press, 1998.

9. Koestler, A. "The Ghost in the Machine", Arkana Books, London, 1969.

10. Koren, Y., Heisel, U., Jovane, F., Moriwaki, T., Pritchow, G., Ulsoy, G. and Brussel, H.V., "Reconfigurable Manufacturing Systems", CIRP, 48 (2), 1999, pp. 527-540.

11. Leitão, P. and Restivo, F. ADACOR: A Holonic Architecture for Agile and Adaptive Manufacturing Control. Computers in Industry 2006; 57(2): 121-130.

12. Leitão, P., Restivo, F. "A Holonic Approach to Dynamic Manufacturing Scheduling”, accepted for publication in Robotics and Computer Integrated Manufacturing, 2008.

13. Manufuture, "Manufuture, A Vision for 2020, Assuring the Future of Manufacturing in Europe", Report of the High-level Group, European Commission, 2004.

14. Massotte, P., "Self-organization: A New Approach to Improve the Reactivity of the Production Systems", Proc. of the International Conference on Emergent Technologies for Factory Automation, 1995, pp. 2332.

15. Mehrabi, M.G., Ulsoy, A.G., Koren, Y. Reconfigurable Manufacturing Systems: Key to Future Manufacturing. Journal of Intelligent Manufacturing 2000; 11: 403-419.

16. Pujo, P., Ounnar, F., "Decentralized Control and Self-organization in Flexible Manufacturing Systems", Proc. of the International Conference on Emergent Technologies for Factory Automation, vol.2, 2001, pp. 659-663.

17. Setchi, R., Lago, N., "Reconfigurability and Reconfigurable Manufacturing Systems - State of the Art Review", 2nd IEEE International Conference on Industrial Informatics 2004, pp. 529-535.

18. Thamarajah, A. A Self-organizing Model for Scheduling Distributed Autonomous Manufacturing Systems. Cybernetics Systems 1998; 29 (5): 461-480.

19. Vaario, J., Ueda, K., "Self-Organisation in Manufacturing Systems", In Japan- USA Symposium on Flexible Automation, 1996, pp. 1481-1484.

2. Vaario, J., Ueda, K., "Biological Concept of Self-organization for Dynamic Shop Floor Configuration", Proc. of Advanced Product Management Systems (APMS'97), 1997, pp. 55-66.

21. Wooldridge, M., “An Introduction to Multi-Agent Systems”, John Wiley \& Sons, 2002. 